\title{
La Gestión del Conocimiento y su incidencia en la Planificación de la ULEAM Extensión Chone
}
Knowledge Management and its impact on the Planning of the ULEAM Chone Extension

\section{Gestão do conhecimento e seu impacto no planejamento da extensão ULEAM Chone}

\author{
Víctor Jama-Zambrano I \\ jviktorz@hotmail.com \\ Katty Zambrano-Alcívar II \\ kattygiza@hotmail.com \\ Jehovana Cornejo-Zambrano III \\ ktyta_cornejo@hotmail.com \\ Lenin Párraga-Zambrano IV \\ lenin_andres_p@hotmail.com
}

Recibido: 30 de enero de 2017 * Corregido: 20 de febrero de 2017 * Aceptado: 20 junio de 2017

\footnotetext{
I. Docente, Universidad Laica Eloy de Manabí, Extensión Chone, Chone, Ecuador.

II. Docente, Universidad Laica Eloy de Manabí, Extensión Chone, Chone, Ecuador.

III. Docente, Universidad Laica Eloy de Manabí, Extensión Chone, Chone, Ecuador.

IV. Docente, Universidad Laica Eloy de Manabí, Extensión Chone, Chone, Ecuador.
} 


\section{Resumen}

Existen muchas teorías y modelos sobre la Gestión del Conocimiento y Planificación. El porqué de nuestra investigación radica en el estudio de estas dos variables que sirvieron para definir el modelo y estilo de Gestión que se está aplicando en la ULEAM Extensión Chone que fue nuestra unidad de análisis. Una vez determinado el modelo se logró identificar el principal objetivo que es: Determinar la planificación como una de las herramientas necesarias en la toma de decisiones.

Conforme a la naturaleza del presente estudio el método empleado es el no experimental de tipo descriptivo. Los resultados obtenidos de la gestión del conocimiento en la ULEAM -CHONE, cuyas dimensiones para este estudio se relacionaron con las sub-dimensiones del Plan de desarrollo Institucional fueron del 89\% en el $2013-2014,80,25 \%$ en el 2015 y el 75\% 2016, en lo cual se puede apreciar una tendencia a la baja en el cumplimiento de este indicador. Por lo tanto, se debe establecer un Modelo de Gestión de Conocimiento que potencie y mejore la planificación de la unidad de análisis investigada.

Palabras clave: Gestión del conocimiento; planificación; modelo y organización. 


\section{Abstract}

There are many theories and models on Knowledge Management and Planning. The reason for our investigation lies in the study of these two variables that served to define the model and style of Management that is being applied in the ULEAM Extension Chone that was our unit of analysis. Once the model was determined, the main objective was identified: Determine planning as one of the necessary tools in decision making.

According to the nature of the present study the method used is the non-experimental descriptive type. The results obtained from the knowledge management in the ULEAM-CHONE, whose dimensions for this study were related to the sub-dimensions of the Institutional Development Plan were $89 \%$ in 2013-2014, 80.25\% in 2015 and 75\% 2016, which shows a downward trend in compliance with this indicator. Therefore, a Knowledge Management Model must be established that enhances and improves the planning of the investigated unit of analysis.

Key words: Knowledge management; planning; model and organization. 


\section{Introducción.}

Analizando el contexto actual se aprecia que en la década de los años 90 la humanidad experimentó un acelerado proceso de transformación social, económica y productiva, las ramas rectoras de esta época se basan en el desarrollo informático, capitales electrónicos, desarrollo de equipos de telecomunicaciones, bases de datos, cibernética, desarrollo de software inteligentes, el láser, entre otros. Este proceso de cambios causó un impacto en los aspectos más esenciales de la vida humana, en donde la transferencia de información y el conocimiento es la característica esencial de la nueva sociedad.

Para este nuevo contexto social se hizo necesario replantear el modelo educativo vigente, porque al encontrarnos en una época distinta a la del siglo $\mathrm{xx}$, los referentes de producción cambiaron y entraron en una profunda crisis. Este cambio de producción al que nos referimos es la elaboración de un producto intangible, es decir, elaborar un producto desde la materia prima tangible ya no era suficiente sino tenía un valor agregado.

Los desafíos para la educación superior en este nuevo milenio deben basarse en la formación de una nueva generación, por lo tanto, se hace necesario comenzar a revisar las propuestas académicas en base a las demandas de la sociedad del conocimiento, replantear nuevas formas de acreditación profesional, las cuales respondan a situaciones de contexto tanto Global como Local (Pensar desde lo global a lo local y viceversa) (Arteaga, 2010).

La decisiva contribución que tienen las universidades en la producción científica, se hace necesario diseñar instrumentos que mejoren su aportación a la humanidad. Por esta razón la Gestión del Conocimiento constituyen una alternativa estratégica para potenciar los resultados de las 
actividades de docencia, investigación y vinculación universitaria en este auge de la sociedad del conocimiento.

Una vez determinada la importancia de esta investigación nos planteamos la siguiente Hipótesis; La aplicación de un Modelo de Gestión de Conocimiento mejorará la Planificación Estratégica de la Universidad Laica Eloy Alfaro de Manabí Extensión Chone.

Por lo que se propuso el siguiente objetivo General: Definir un Modelo de Gestión de Conocimiento en la Planificación Estratégica de la Universidad Laica Eloy Alfaro de Manabí Extensión Chone, el cual se cumplió una vez que se ejecutaron los objetivos secundarios como eran:

- Determinar la planificación como una de las herramientas necesarias en la toma de decisiones.

- Definir los niveles de organización en la gestión del conocimiento en orden de jerarquización de la ULEAM Chone.

- Diseñar una propuesta de un Modelo de Gestión de Conocimiento para la Planificación Estratégica de la ULEAM Chone

Estos objetivos se comprobarán mediante los procesos de validación de hipótesis específicas:

- La Planificación en la Gestión del conocimiento interviene como una de las herramientas necesarias en la toma de decisiones de la ULEAM Chone

- El nivel de organización en la gestión del conocimiento afecta los mandos en orden de jerarquización de la ULEAM Chone.

- La propuesta de un nuevo Modelo de Gestión de Conocimiento mejorará la Planificación Estratégica de la ULEAM Chone. 
Las implicaciones teóricas y prácticas de este estudio se basaran una rápida revisión de las definiciones dadas acerca de la «Gestión del Conocimiento» pone en evidencia un cierto caos conceptual, atribuible, entre otras causas, a la relativa juventud de la disciplina, que conlleva la ausencia de un cuerpo doctrinal sólido y estructurado, y a la diversidad de disciplinas de origen de los autores que abordan la temática, por lo que podemos definir qué gestión del conocimiento, es el conjunto de procesos sistemáticos (identificación y captación del capital intelectual; tratamiento, desarrollo y compartimiento del conocimiento; y su utilización) orientados al desarrollo organizacional y/o personal y, consecuentemente, a la generación de una ventaja competitiva para la organización y el individuo. (Rodríguez, 2006).

Según Balmori \& Schmelke, (2012): Gestión es hacer que las cosas sucedan en cualquier área del conocimiento; sin embargo, cuando se dice gestión del conocimiento se está hablando de creación, transferencia, almacenamiento, aplicación y uso del conocimiento mismo; lo que se debe considerar es un elemento muy importante: el capital intelectual. En cualquier área del conocimiento, y más aún en la educación donde se encuentra involucrado el capital intelectual.

La gestión de conocimiento en las Universidades, en un sentido genérico tiene como fines la creación o generación de conocimiento humano y tecnológico, mediante sus actividades de estudio e investigación científico-técnica, y la transmisión del conocimiento individual y colectivo, mediante sus actividades docentes y formativas de pregrado y postgrado, así como las de extensión o difusión de la cultura a la sociedad en general. (Martínez, Peñalver \& Salamanca, 2007). 


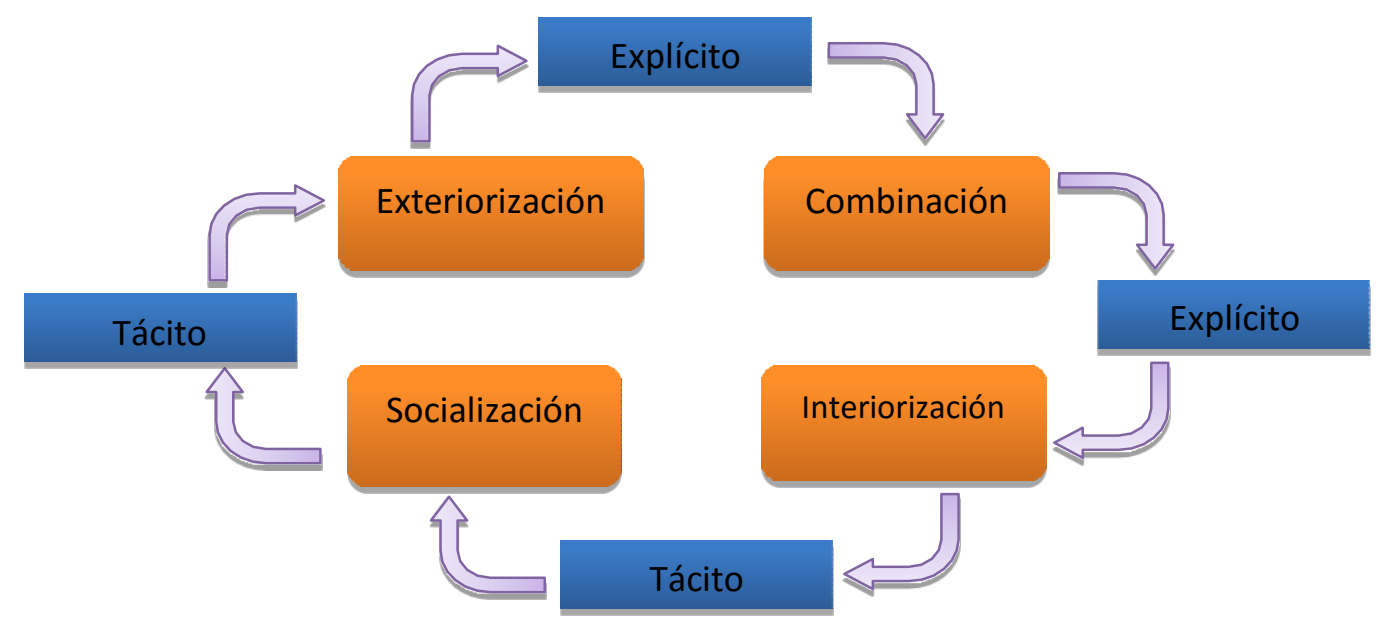

Figura 1: Proceso de conversión del conocimiento en la organización

Fuente: Nonaka y Takeuchi (1995)

\section{Importancia del Problema}

De acuerdo a la investigación realizado por ARTURO Rodríguez CASTELLANOS Andrés ARAUJO DE LA MATA JAVIER URRUTIA Gutiérrez sobre "La gestión del conocimiento científico técnico en la universidad" de la Universidad del País Vasco (UPV/EHU -2012), actualmente, las universidades se enfrentan a aumentos en sus necesidades de financiación, cuando el criterio predominante es que los poderes públicos tengan una menor presencia en la misma. Por otra parte, algunos investigadores indican que la Universidad parece estar perdiendo posiciones como centro de generación de conocimiento, el cual, como recurso en si mismo, está siendo objeto de creciente atención por parte de las empresas y los gobiernos, considerándosele el recurso clave en la consecución de ventajas competitivas. 
En estas circunstancias, estimamos que la Universidad debe hacer mayores esfuerzos en orientar su investigación a la aplicación del conocimiento científico- técnico, en colaboración con las empresas y las administraciones públicas, como indica el modelo de la triple hélice de Etzkowitz y Leydesdorff.

En este trabajo se presenta también cómo se afronta en la Universidad del País Vasco/Euskal Herriko Unibertsitatea la gestión del conocimiento científico- técnico, así como un proyecto de investigación cuya finalidad consiste en la búsqueda de un modelo de gestión de este conocimiento en el contexto descrito.

En conclusión, si la gestión del conocimiento está recibiendo gran atención por parte tanto de técnicos como de gestores de empresas y organizaciones en general, por considerar al conocimiento como el factor distintivo de la empresa en el logro de ventajas competitivas; y también por parte de los gobiernos, por considerar que es un factor de vital importancia en el crecimiento económico y el bienestar de un país, la Universidad no puede quedar ajena a esta corriente, desde el momento en que su función es la de producir y difundir conocimiento. Y debe sumarse a ella tanto si se considera a la Universidad una institución de servicio público, como desde el punto de vista de ser una organización particular Como institución que realiza un servicio público, lo debe hacer para mantener su posición dominante en la generación de conocimiento, de forma que no se cumplan las predicciones de algunos autores como Gibbons et al (1994) sobre que la Universidad dejará de ser el principal foco de producción de conocimiento científico-técnico. Es cierto que el conocimiento ya no se genera sólo en la Universidad, sino que cada vez lo hace más en otras entidades, como centros tecnológicos, consultorías, empresas, etc., y que además se tiende a producirse en contextos de aplicación; pero la Universidad cuenta todavía con fuertes ventajas competitivas, como por ejemplo 
una mayor eficiencia en costes, elevada creatividad y tradición, aunque podrá perderlas de no adaptarse a la nueva realidad técnica y social.

Para comprender el concepto de "gestión del conocimiento" aplicado en la Universidad debemos reconocer que el mismo involucra por lo menos estas dimensiones: a) una teoría del conocimiento; b) una teoría de la acción; c) una teoría institucional; d) una política del conocimiento; e) una metodología para transmitir y aplicar los conocimientos.

En el ámbito universitario nos encontramos con una organización donde el conocimiento no es un medio sino un fin. La universidad produce nuevos conocimientos (investigación), forma profesional y transfiere saberes científicos o técnicos para resolver problemas de la sociedad. Además, la organización universitaria divide los saberes de acuerdo a criterios teóricos o profesionales y se somete a evaluaciones institucionales, científicas y técnicas para acreditar la transmisión de conocimientos.

"La enseñanza superior trabaja con una teoría del conocimiento implícita: aquella que acepta que en cada disciplina o carrera se apliquen "modelos de conocimiento" diferentes. Esto vale entre las diferentes disciplinas, pero también al interior de un mismo campo disciplinario" (Pérez Augusto 2011)

\section{Modelo seci aplicado a la universidad}

El modelo de gestión de conocimiento propuesto, está basado en el modelo SECI, para ello se identifican las actividades universitarias involucradas en cada una de las etapas del modelo. La siguiente tabla, describe la propuesta de modelo de gestión de conocimiento aplicado a la universidad boliviana. 


\begin{tabular}{|c|c|}
\hline $\begin{array}{l}\text { Etapas del modelo } \\
\text { SECI }\end{array}$ & Actividades Universitarias \\
\hline $\begin{array}{l}\text { Socialización } \\
\text { (tácito a tácito) }\end{array}$ & $\begin{array}{l}\text { - Realización de congresos o seminarios conjuntos entre } \\
\text { universidad y empresa. } \\
\text { - Realización de prácticas en empresas. } \\
\text { - Implementación de prácticas de estudiantes. }\end{array}$ \\
\hline $\begin{array}{l}\text { Externalización } \\
\text { (tácito a explícito) }\end{array}$ & $\begin{array}{l}\text { - Investigación en asociación con las Instituciones públicas o } \\
\text { privadas. } \\
\text { - Asociación con las Instituciones públicas o privadas para } \\
\text { educación y entrenamiento. }\end{array}$ \\
\hline $\begin{array}{l}\text { Combinación } \\
\text { (explícito a explícito) }\end{array}$ & $\begin{array}{l}\text { - Creación de mapas de aprendizaje continuo. } \\
\text { - Creación de equipos de investigación interdisciplinarios. } \\
\text { - Creación de centros para la transferencia de tecnologías a la } \\
\text { industria. } \\
\text { - Conformación de juntas y consejos consultivos universidad- } \\
\text { - Instituciones públicas o privadas. } \\
\text { - Creación de bases de conocimiento. }\end{array}$ \\
\hline $\begin{array}{l}\text { Interiorización } \\
\text { (explícito -tácito) }\end{array}$ & $\begin{array}{l}\text { - Difusión vía sistema de información ejecutivo vía creación de } \\
\text { sitios web dinámicos. } \\
\text { - Creación de redes de trabajo colaborativo. } \\
\text { - Creación de una cultura que aliente el intercambio de } \\
\text { conocimientos. }\end{array}$ \\
\hline
\end{tabular}

Tabla $N^{\circ}$ 1.- Modelo SECI aplicado a la universidad

Fuente: Elaborado por Juan Carlos Huanca Guanca.

\section{Metodología}

Es una Investigación no Experimental que está enfocado principalmente a buscar la profundidad a los datos y la información, a la separación de los mismos (dispersión) a la riqueza interpretativa, a la contextualizada del entorno, los detalles y experiencias únicas que permitan aportar puntos de vista natural y holístico de los fenómenos, así como aportar la flexibilidad que corresponda, tratando de potenciar el desarrollo del conocimiento y la resolución de problemas dentro de los fenómenos empíricos o formalizados del mundo que nos rodea (la realidad) que se investiga 
Para la recolección y el procesamiento de la información, se hizo una tabla de indicadores asociados relacionando la Gestión del Conocimiento con la planificación de acuerdo a los informes anuales de rendición de cuentas de la IES. Además, se aplicó una ficha de datos que permitió obtener la información correspondiente a los indicadores de gestión del conocimiento y planificación.

Toda la información registrada en las fichas de recolección de datos fue doblemente digitada, haciendo uso de la hoja de cálculo Excel y el manejo del software SPSS, con el objeto de dar respuesta al problema y la hipótesis de investigación.

Una vez controlada la calidad de los datos se procedió a realizar un análisis descriptivo de nuestros resultados, resumiendo nuestras variables cualitativas según sus cuadros y gráficos de frecuencia absoluta y relativa. Para tal efecto, en el caso de las variables cuantitativas a priori, se verificará la normalidad de su distribución de forma gráfica y estadística.

\section{Variable Independiente ( $x$ )}

X = La gestión del conocimiento

En el caso de la variable independiente se tienen dos indicadores.

$\mathrm{X} .1$ = Conocimiento Tácito.

X.2 = Conocimiento Implícito

\section{Variable Dependiente (y)}

Y = Planificación.

La variable dependiente posee dos indicadores.

Y1 = Plan Estratégico de Desarrollo Institucional. 
Y2 = Plan Operativo Anual.

\section{Conocimiento Tácito en la Planificación (X1)}

Comisiones de Extensión 1.

Dónde: $\quad \mathrm{X} 1=\quad$ Departamentos Centrales.

\section{Conocimiento Explicito en la Planificación (X2)}

Planes Operativos Anuales.

Dónde: $\quad \mathrm{X} 2=$

Plan Estratégico de Desarrollo Institucional.

Tabla Indicadores Asociados a la Gestión del Conocimiento

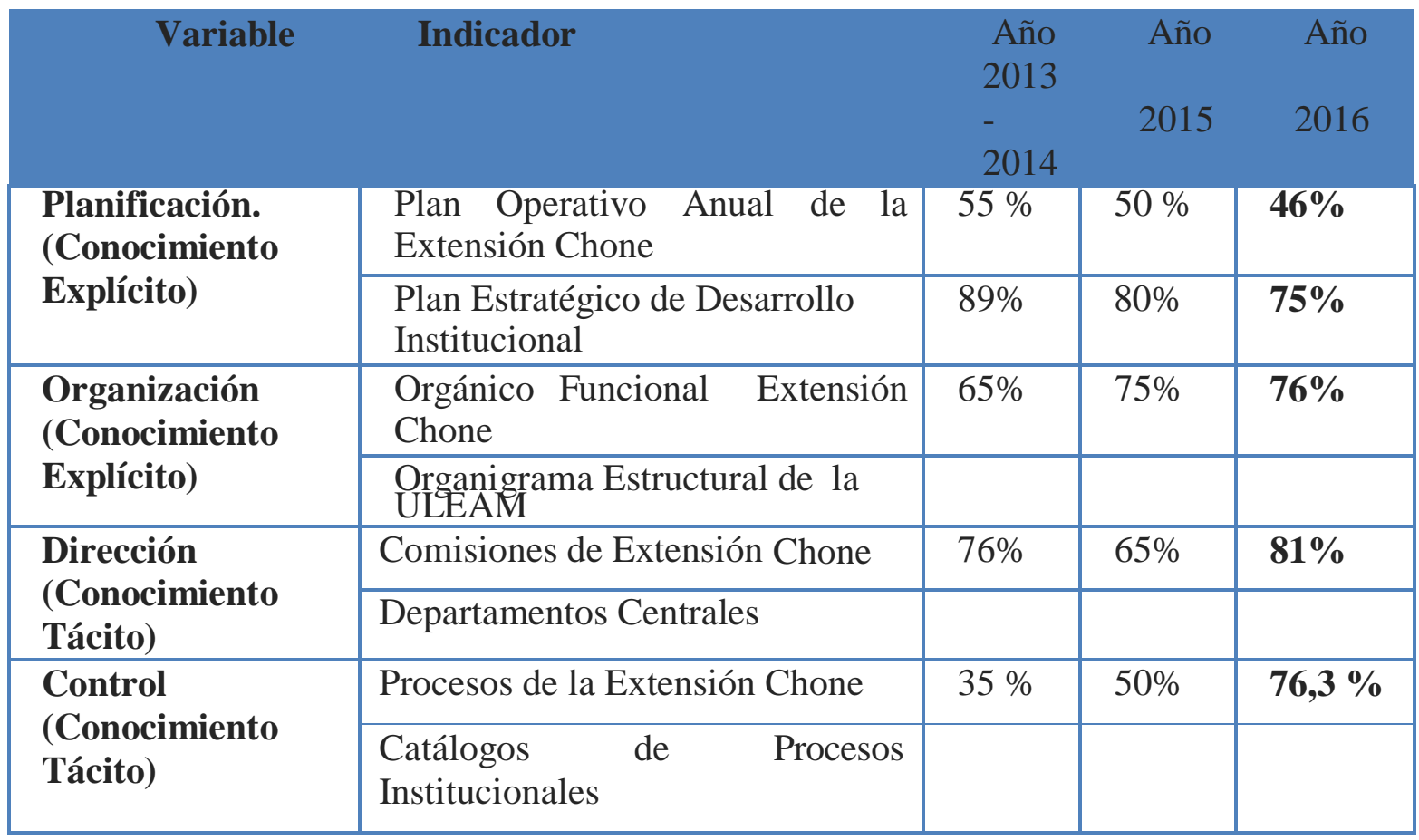




\section{Resultados}

Los resultados obtenidos en esta investigación se basaron en los siguientes objetivos:

- Determinar la planificación como una de las herramientas necesarias en la toma de decisiones.

Figura 1

Cumplimiento del Plan de Desarrollo Institucional

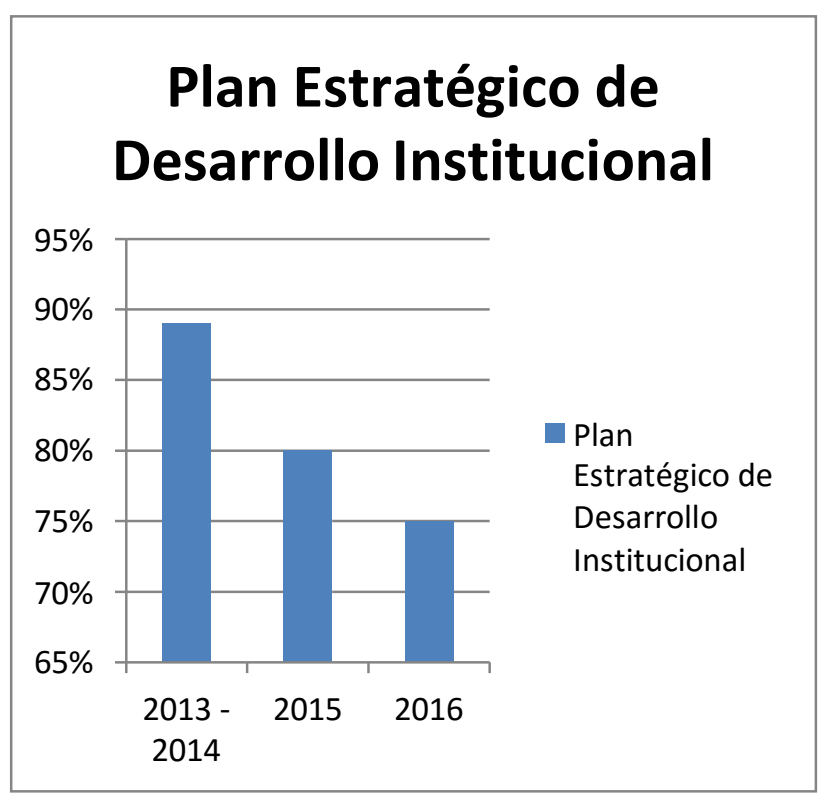

Fuente: Rendición de Cuentas ULEAM.
Figura 2

Cumplimiento del Plan Operativo Anual de la Extensión Chone

\section{Plan Operativo Anual de la Extensión Chone}

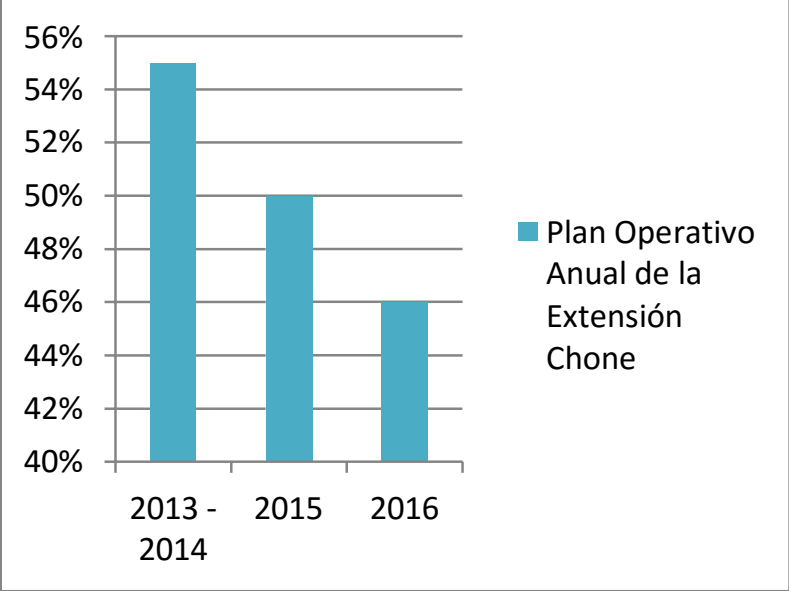

Fuente: Rendición de Cuentas ULEAM.

Estos objetivos se comprobarán mediante los procesos de validación de hipótesis específicas:

Se comprueba la H1. Que la Planificación en la Gestión del conocimiento interviene como una de las herramientas necesarias en la toma de decisiones de la ULEAM Chone 
Relacionar los niveles de organización en la gestión del conocimiento en orden de jerarquización de la ULEAM Chone.

Figura 3

Cumplimiento de los niveles de Organización en la Planificación de la Extensión Chone.
Figura 4

Cumplimiento del Plan Operativo Anual de la Extensión Chone

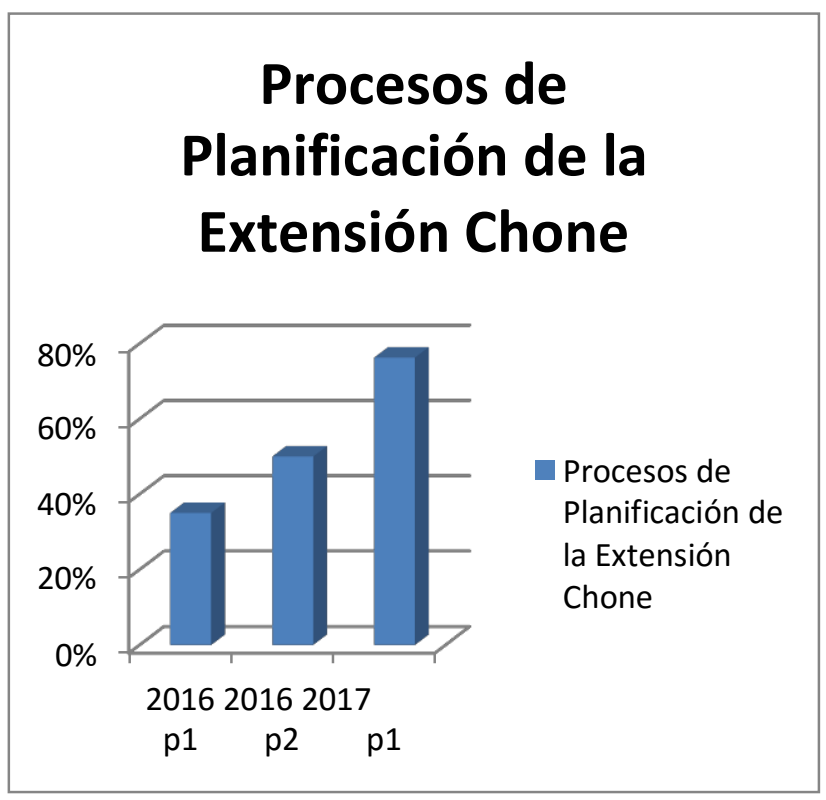

Fuente: Rendición de Cuentas ULEAM.

\section{Organización de comisiones de Extensión Chone}

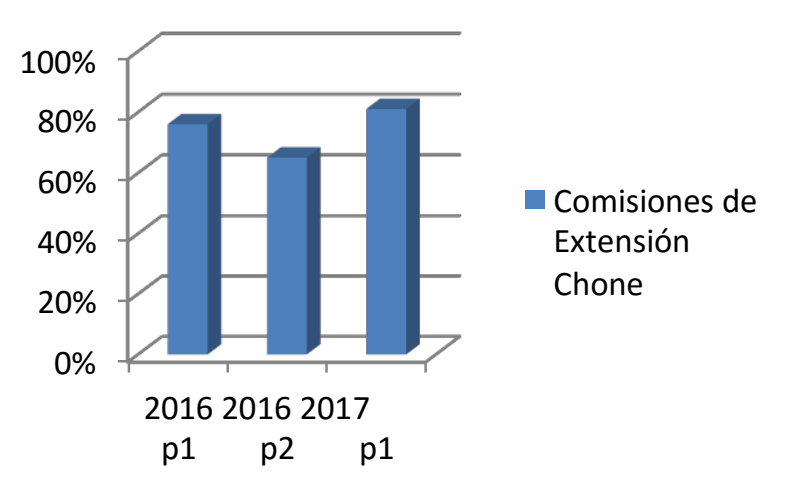

Fuente: Rendición de Cuentas ULEAM.

H2. Se comprueba la relación existente entre los niveles de organización en la gestión del conocimiento y los mandos en orden de jerarquización de la ULEAM Chone. 
- Diseñar una propuesta de un Modelo de Gestión de Conocimiento para la Planificación Estratégica de la ULEAM Chone.

\section{Figura 5:}

Modelo de Gestión de Conocimiento Aplicado a la Universidad Pública en el Perú Fuente: (Díaz, 2003)

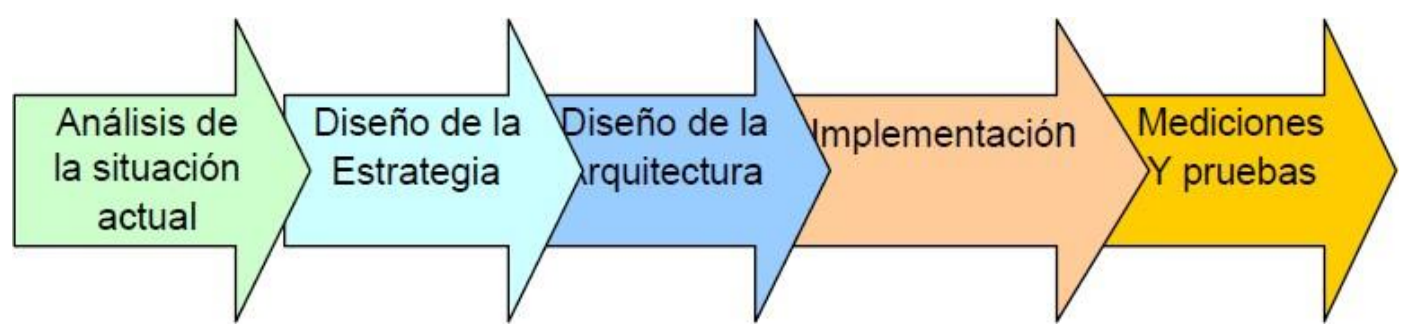

Figura 6: Propuesta de Modelo de Gestión de Conocimiento

Aplicado a la ULEAM-CHONE

Fuente: (Propia)
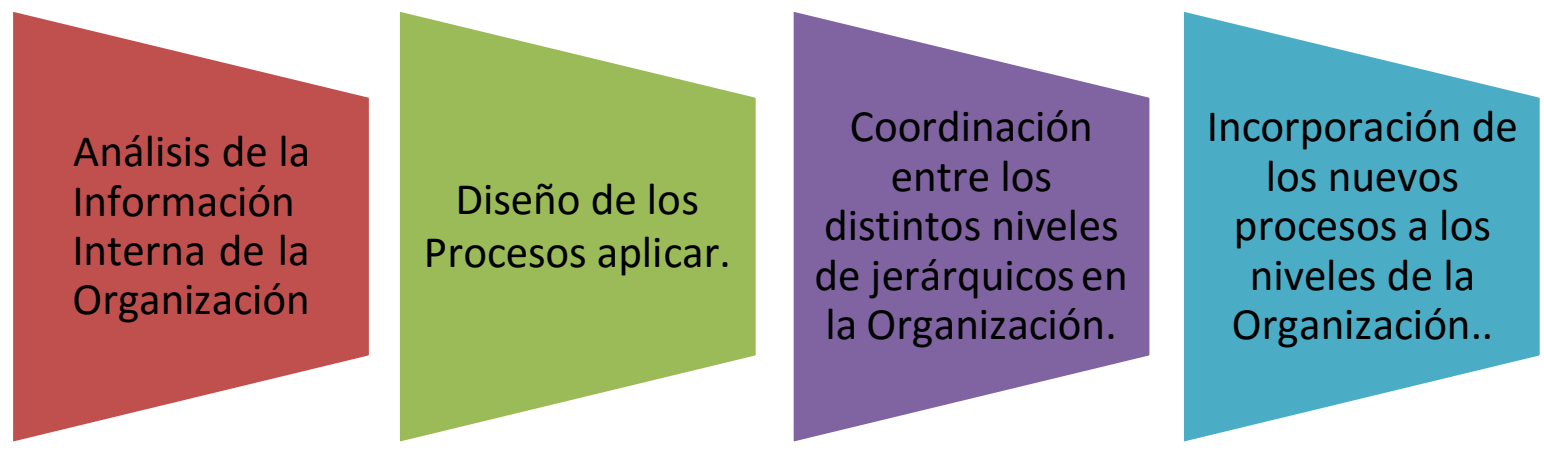


\section{Conclusión}

La aplicación del modelo de gestión de conocimiento, consiste en la aplicación periódica por gestión de los indicadores carrera, que culmina con un informe de gestión que describe la situación actual del programa con las respectivas recomendaciones de mejora a ser abordadas para la siguiente gestión identificando prioridades a incorporar en el plan operativo anual de la siguiente gestión, este proceso es gradual y permanente definido para cada gestión metas específicas con el compromiso de las autoridades.

Con el desarrollo del presente trabajo, se llegó a analizar la situación de la extensión universitaria en relación a la incorporación de modelos de gestión de conocimiento. Su aplicación varía del modelo SECI, observando que en ciertas universidades de Latinoamérica se realizan esfuerzos en la identificación de indicadores de Gestión, Planificación, Organización y Control.

En el trabajo, se llega a establecer indicadores de Gestión del Conocimiento y Planificación que rescatan los trabajos previos desarrollados en universidades de otros países y se contextualiza con las normativas vigentes en el país con los modelos e indicadores utilizados para la acreditación de programas y/o carreras vigentes. El trabajo llega a definir además un análisis de las actividades de la extensión universitaria que se vinculan directamente al modelo SECI de gestión de conocimiento junto con la planificación que viabilizan el ciclo del conocimiento.

El trabajo es un gran aporte para la extensión universitaria en Chone, al ser uno de los primeros trabajos desarrollados en este ámbito y brinda un modelo para la transformación de la planificación basada en la Gestión del conocimiento. 


\section{Referencias}

ARRAUT \& Gazabón, (2010) "Modelo DECREXA para la gestión de conocimiento" trabajo elaborado por profesores de los programas de Ingeniería Industrial y Administración de Empresas de la Universidad Tecnológica de Bolívar en Cartagena de Indias.

CANALS, A, (2003). La Gestión del Conocimiento. http://www.uoc.edu/dt/20251/index.html

CALDERÓN C. Mónica (2013), "El capital intelectual de la investigación en los docentes de posgrado de una universidad privada de Lima", PUCP.

DAVENPORT, T. \& PRUSAK, L. (2000). Working Knowledge how Organizations Manage what they know. Boston: Harvard business school.

DÍAZ M. Jorge (2003) trabajo sobre cómo "construir un modelo para la gestión del conocimiento en una realidad educativa universitaria del país, a partir de los procesos de autoanálisis institucional y la construcción de un sistema de indicadores de la calidad educativa...”.

EDVINSSON, L. y Malone, M. (1998). El Capital Intelectual: Cómo identificar y calcular el valor inexplorado de los recursos intangibles de su empresa. (2nd ed., pp. 30-48). Bogotá: Norma.

EUROFORUM 1998. Proyecto Intelect. Medición del capital intelectual. Madrid: Euroforum. 200 p.

López, M. (2012). Gerencia: capital intelectual y sus competencias en instituciones educativas. Observatorio Laboral Revista Venezolana, 5(9), 109-127.

MARSHALL, C., Prusak, L. y Shpilberg, D. (1996). "Financial risk and the need for superior knowledge management”. En: L. Prusak (ed.). Knowledge in organizations. Boston, EUA: Butterworth-Heinemann, pp. $227-251$.

MARTÍNEZ, F., Peñalver, A., Salamanca, J. (2007). Gestión estrátegica del conocimiento. Universidad de Cantabria.

MOSQUERA Luz. (2011) investigación en la Facultad de Administración Manizales de la Universidad Nacional de Colombia sobre "Gestión del Capital Intelectual de las Instituciones Educación Superior. caso Universidad Nacional de Colombia- Sede Manizales"

RAMÍREZ C. Yolanda (2011). Investigación sobre "Capital Intelectual en las Instituciones de Educación Superior" de la Universidad De Castilla La Mancha.

Revista Virtual Universidad Católica del Norte". No.27, (mayo - agosto de 2009, Colombia), acceso: [http://revistavirtual.ucn.edu.co/], ISSN 0124-5821.

TOFFLER A. (1980) tercera ola. Plaza \& janes. s.a. editor. Bogotá. 
TOPETE, C., Bustos, E., Bustillos, E. (enero-junio, 2012). Gestión del conocimiento para promover la productividad académica de los institutos tecnológicos en la sociedad del conocimiento. Sinéctica, 38. Recuperado de http://www.sinectica.iteso.mx/index.php?cur=38\&art=38_06.

VALENCIA Cárdenas, M., Estrada Muñoz, J., Bedoya Botero, I. B., Tuttle Ospina, S., \& Gaviria Berrio, F. (2013). Indicadores del capital intelectual en el área de ingenierías de una universidad. Revista Ciencias Estratégicas, 22(30), 285-298.

VALHONDO D. (2003). Gestión del conocimiento del mito a la realidad ediciones Díaz de santos, Madrid, pág., 51 